\title{
As capacidades dinâmicas de TI e a sustentação do ERPII
}

\section{Antônio Cesar Polo}

Mestre em Controladoria e Contabilidade Estratégica pela Fundação Escola de Comércio Álvares Penteado, São Paulo, Brasil

acesarpolo@gmail.com

Claudio Parisi

Doutor em Controladoria e Contabilidade pela Universidade de São Paulo. Professor pesquisador da Fundação Escola de Comércio Álvares Penteado e Coordenador do Programa de Mestrado em Ciências Contábeis da FECAP. Professor pesquisador da Fundação Escola de Comércio Álvares Penteado e Coordenador do Programa de Mestrado em Ciências Contábeis da FECAP, São Paulo, Brasil claudio.parisi@uol.com.br

César Augusto Biancolino

Doutor em Controladoria e Contabilidade pela Universidade de São Paulo. Professor do Departamento de Administração da Universidade CEUMA, Maranhão, Brasil. biancolino@gmail.com

\section{Editor Científico: José Edson Lara}

Organização Comitê Científico

Double Blind Review pelo SEER/OJS

Recebido em 23.08.2017

Aprovado em 20.10.2017

\section{(c) (i) (8)}

Este trabalho foi licenciado com uma Licença Creative Commons - Atribuição - Não Comercial 3.0 Brasil 


\title{
Resumo
}

Este trabalho procurou identificar a influência das capacidades dinâmicas na sustentação dos sistemas de gestão empresarial (ERPII). De acordo com a literatura, as capacidades dinâmicas influenciam positivamente o valor de uso dos sistemas de informação e, por conseguinte, promovem o aumento do desempenho organizacional. Em um enfoque geral, elas evitam a dissipação dos investimentos feitos no ERPII. Para viabilizar o estudo, um modelo conceitual foi criado, com o objetivo de articular as variáveis relativas aos dois constructos. Com base neste modelo, foi realizada uma pesquisa de campo de natureza quantitativa, com 374 colaboradores de áreas de tecnologia da informação de empresas de grande porte que atuam no contexto brasileiro. Os resultados foram analisados por meio de métodos de estatística descritiva e regressão linear múltipla. A pesquisa contribui para o desenvolvimento dos estudos sobre capacidades organizacionais como impulsionadoras da vantagem competitiva.

Palavras-chave: ERPII; Capacidades dinâmicas; Vantagem Competitiva; Estratégia.

\section{Dynamic IT capabilities and the support of ERPII}

\begin{abstract}
This work investigates the influence of dynamic capabilities in ERPII systems. According to the literature, dynamic capabilities positively influence the value of use of information systems and therefore improve organizational performance. In a general approach, they avoid the dissipation of investments made in ERPII. To make the study viable, a conceptual model was created with the objective of articulating the variables related to the two constructs. Based on this model, a field research of a quantitative nature was created, with 374 employees in information technology areas of large companies, who operate in the Brazilian context. The results were analyzed by means of descriptive statistics and multiple linear regression. The research contributes to the development of studies on organizational capabilities as drivers of competitive advantage.
\end{abstract}

Key-words: ERPII; Dynamic Capabilities; Competitive Advantage; Strategy.

\section{Las capacidades dinámicas de TI y la ayuda del ERPII}

\section{Resumen}

Este trabajo buscó identificar la influencia de las capacidades dinámicas en la sustentación de los sistemas de gestión empresarial (ERPII). De acuerdo con la literatura, las capacidades dinámicas influencian positivamente el valor de uso de los sistemas de información y, por consiguiente, promueven el aumento del desempeño organizacional. En un enfoque general, evitan la disipación de las inversiones 
realizadas en el ERPII. Para viabilizar el estudio, un modelo conceptual fue creado, con el objetivo de articular las variables relativas a los dos constructos. Con base en este modelo, se creó una encuesta de campo de naturaleza cuantitativa, con 374 colaboradores de áreas de tecnología de la información de empresas de gran porte, que actúan en el contexto brasileño. Los resultados se analizaron mediante métodos de estadística descriptiva y regresión lineal múltiple. La investigación contribuye al desarrollo de los estudios sobre capacidades organizacionales como impulsoras de la ventaja competitiva.

Palabras clave: ERP II; Capacidades dinámicas; Ventaja competitiva; Estrategia.

\section{Introdução}

Os sistemas de gestão empresarial são soluções evolutivas e devem promover benefícios às empresas no cenário atual de negócios. Dentre eles, destacam-se a capacidade de tratamento analítico da informação, suporte à tomada de decisão e integração com a cadeia de valor (fornecedores, clientes, parceiros de negócios). Este modelo de sistemas tem o nome de ERP II (enterprise resource planning) (Moller, 2005; Norton, 2013).

Os esforços e valores financeiros empregados na implementação e sustentação do ERP II não são pequenos. Portanto, os acionistas têm a expectativa de mensurar os benefícios e retornos sobre esses investimentos. Todavia, observase na literatura uma falta de consenso quanto à metodologia de determinação do ROI para tais projetos. Por outro lado, empresas que não exercerem o controle de suas operações mediante o uso desta tecnologia podem estar em desvantagem competitiva (Souza \& Zwicker, 2007; Jain, 2010).

Esta problemática abre espaço para que se observem os investimentos dos ERP II sob aspectos intangíveis, em detrimento de um enfoque unicamente baseado em indicadores monetários. Nesse contexto, seus benefícios indiretos são obtidos quando o ERP II torna-se impulsionador do desenvolvimento das capacidades organizacionais ao longo do tempo de uso do sistema (Borges, Parisi, \& Gil, 2005; Lu \& Jinghua, 2012).

O estudo do impacto das capacidades organizacionais no desempenho das empresas tem seus fundamentos na teoria da Visão da Firma Baseada em Recursos, ou Resources Based View (RBV), que analisa a posse e articulação dos recursos estratégicos definidos como valiosos, raros, difíceis de imitar e não substituíveis (Barney \& Clark, 2007). 
Todavia, a RBV apresenta inadequações em ambientes com altas taxas de mudanças. Esse contexto dificulta a obtenção de ganhos de competitividade baseados na simples posse de um feixe diferenciado de recursos. Nesses ambientes, pode não haver tempo e racionalidade suficientes para a reposição adequada dos recursos. Para que o diferencial competitivo se sustente, é necessário que a companhia desenvolva capacidades de integração, renovação e recombinação dos recursos existentes. Tais capacidades são definidas na literatura como capacidades dinâmicas (Ambrosini \& Bowman, 2009).

Neste contexto, tem-se a questão principal de pesquisa: Qual é o relacionamento entre as Capacidades Dinâmicas de TI e a sustentação do ERP II na fase de pós-implementação? Visando tratar esta problemática, adotou-se como objetivo principal criar um modelo analítico para articular as variáveis relativas aos dois constructos, a saber: o relacionamento das capacidades dinâmicas de TI com a percepção de valor de uso do ERP II em sua fase de sustentação.

\section{Referencial Teórico}

\subsection{ERP II}

A partir da evolução dos aplicativos ERP implementados em larga escala a partir da década de 1990, as primeiras versões dos aplicativos ERP II passaram a incorporar funcionalidades nativas de outros aplicativos. Os ERP II mais recentes têm sido desenvolvidos tanto se utilizando de novas funcionalidades nativas, também originárias primariamente de outros aplicativos, como também se utilizando dos serviços orientados a objeto, o que potencializa o conceito do ERP II como um sistema multifuncional, voltado para o front office (ou comunicação com governo, clientes e fornecedores), resguardando, no entanto, toda a capacidade de registrar e consolidar as informações de back office, ou internas à organização.

De acordo com Moller (2005), o ERP II inclui seis elementos que permeiam o negócio, os aplicativos e a estratégia de TI da organização. Para Moller (2005, p. 483), as seis características básicas dos aplicativos ERP || são: "(1) as funções do ERP II; (2) o domínio do negócio; (3) as funcionalidades referenciadas a este domínio; (4) os tipos de processos requeridos por essas funcionalidades; (5) a 
arquitetura de sistema que possa suportar estes processos e (6) a forma pela qual os dados são tratados por essa arquitetura" .

Segundo esta definição, com exceção da arquitetura, essas características reunidas caracterizam o ERP II como a expansão do ERP tradicional e, como conclusão, pode-se definir o ERP II como uma solução composta pela conjunção de funcionalidades do ERP tradicional e do comércio eletrônico colaborativo na cadeia de fornecedores, que estão se consolidando à medida que os aplicativos ERP clássicos estejam sendo ou forem substituídos pelos aplicativos ERP II, de forma gradual (Moller, 2005). Esta definição pode ser representada graficamente pela Figura 1.

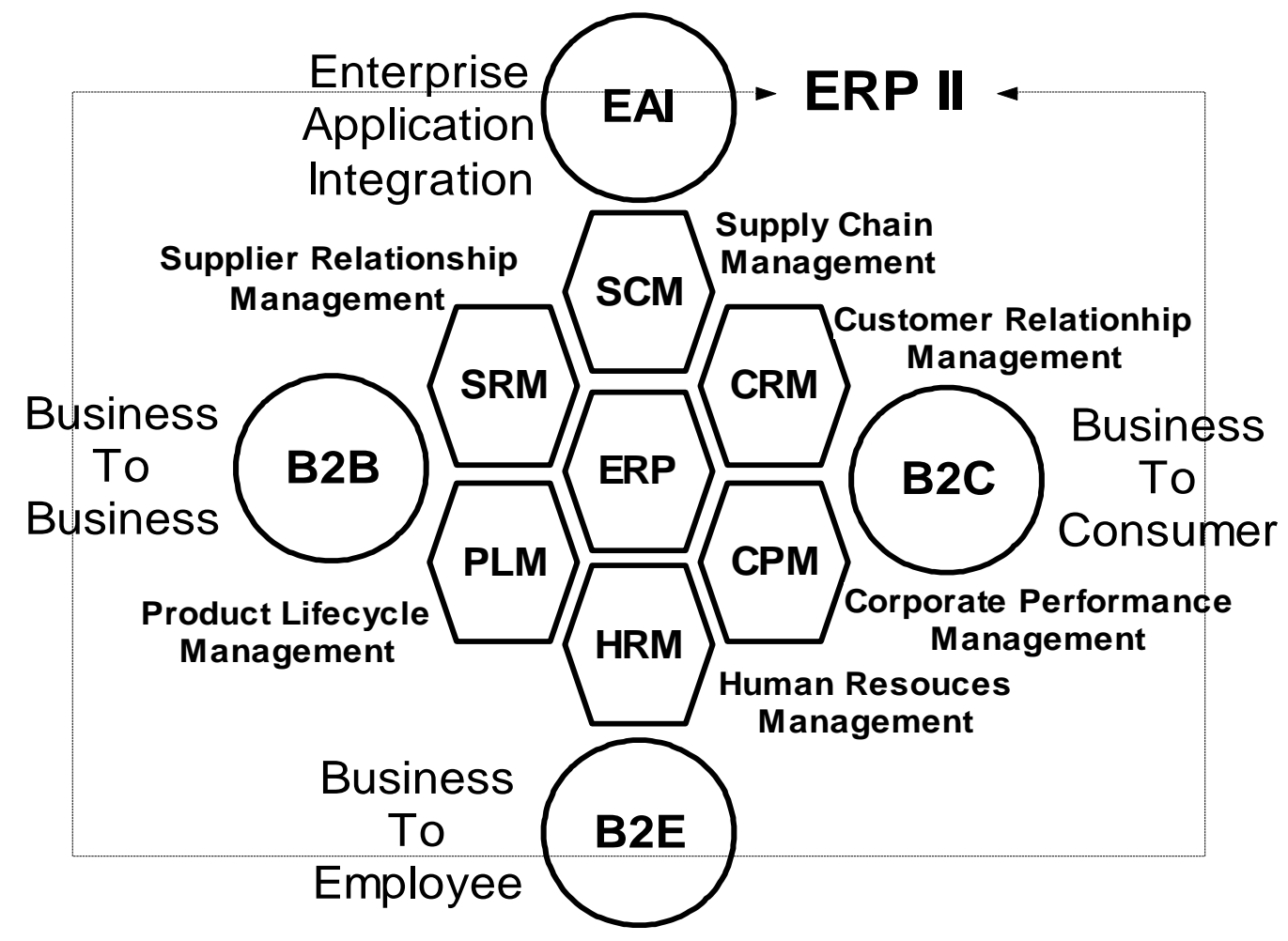

Figura 1 - Estrutura conceitual do ERP II

Fonte: Moller, C. (2005). ERP II: A conceptual model for next-generation enterprise systems. Journal of Enterprise Information Management, 18(4), 483-497.

Segundo essa abordagem, a estrutura de um aplicativo ERP II pode ser visualizada como um sistema composto por três camadas que realizam a gestão dos processos da organização (BPM) - que podem ou não estarem inclusos ao ERP tradicional - e formam o núcleo da estrutura do aplicativo ERP II. Este fato, como dito anteriormente, justifica-se pelo fato de o ERP clássico possuir a característica de 
sistema integrador e centralizador dos dados provenientes das transações monetárias da organização.

De acordo com as explanações anteriores, as quais afirmam que o sistema ERP tradicional está "recebendo" novas funcionalidades, deslocando-se desta forma no eixo das funcionalidades do back office para o front office, pode-se afirmar que a construção do conceito de ERP II se sustenta com base nas seguintes premissas:

a) Existe a necessidade de as empresas participantes de uma mesma cadeia de suprimentos comunicarem-se entre si de uma forma cada vez mais ágil e efetiva. A globalização, a competitividade e a constante procura pela otimização de processos e redução de custos criam constantemente a necessidade de integração entre as empresas;

b) $O$ avanço da tecnologia da informação, especialmente com o advento da arquitetura de serviços SOA (Services Oriented Architecture), está permitindo que sistemas de diferentes naturezas se integrem de forma nunca antes possível;

c) Devido ao fato do ERP clássico conter em sua base de dados informações de diversas naturezas e de diferentes granularidades, o mesmo ocupa naturalmente o "centro" de um novo conjunto de aplicativos denominado ERP II.

De outra forma, é correto afirmar que, sem a presença das funcionalidades básicas de um ERP convencional, não se pode construir o conceito de um ERP mais amplo, direcionado ao ambiente externo, já que as características fundamentais do ERP clássico (centralização e consolidação das transações monetárias em uma base única de dados) continuam a formar o pilar principal do ERP II, ainda que este comporte uma série de novas funcionalidades de forma comparada com as primeiras versões dos aplicativos ERP, conforme ilustra a Figura 2. 


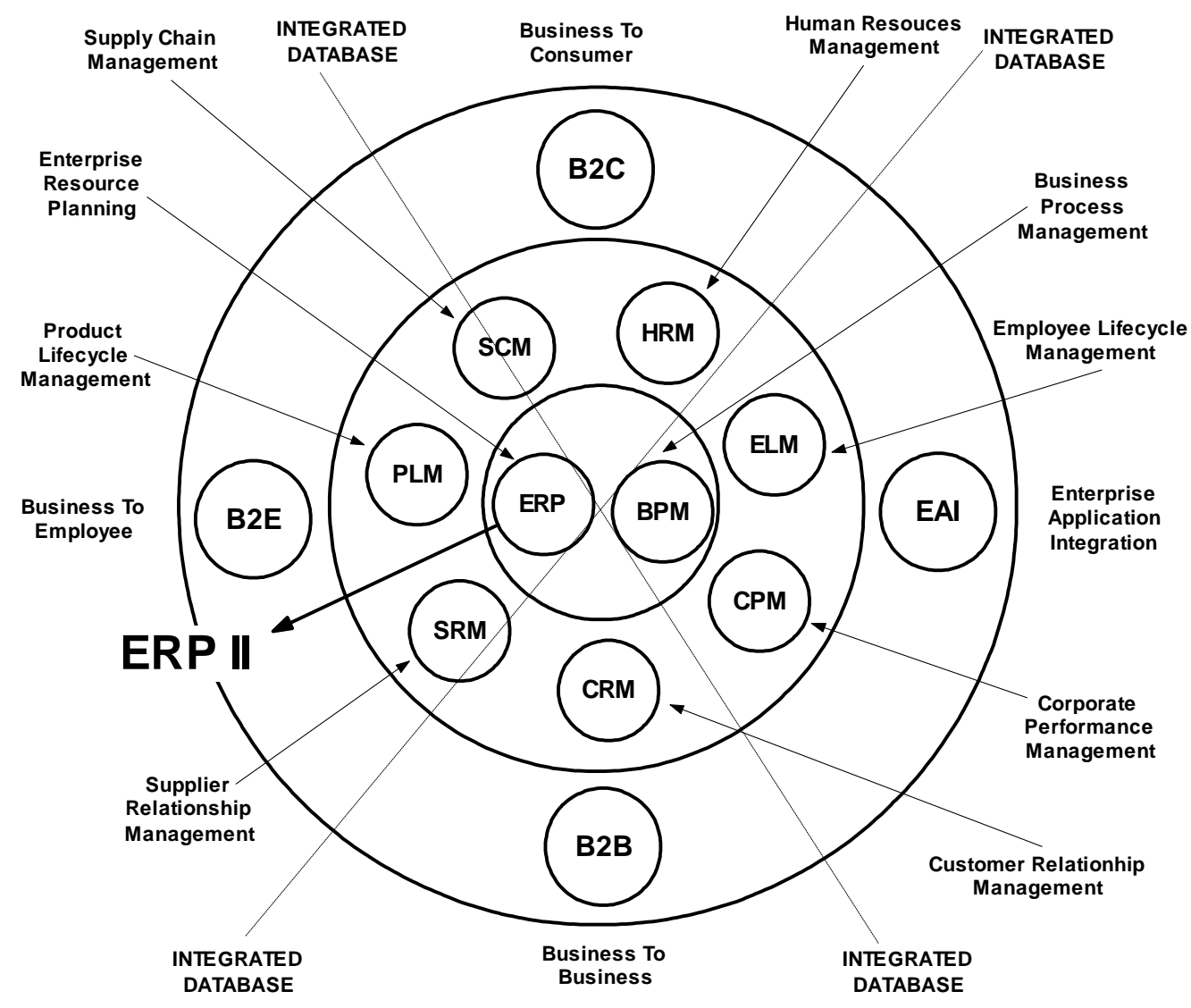

Figura 2 - As camadas estruturais do aplicativo ERP II

Fonte: Biancolino, C. A. (2010). Valor de uso do ERP e gestão contínua de pós implementação: estudo de casos múltiplos no cenário brasileiro. (Tese de doutorado. Universidade de São Paulo, São Paulo, SP, Brasil).

A segunda camada da estrutura do aplicativo ERP II é composta pelas funcionalidades dos sistemas ditos organizacionais ou corporativos. De acordo com a estruturação sugerida por Moller (2005), esses sistemas possuem as seguintes características, expressas pela Tabela 1:

Tabela 1

Características dos sistemas corporativos associados ao conceito de ERP II

\begin{tabular}{c|c|l|l}
\hline Sigla & Definição & \multicolumn{1}{|c}{ Tradução } & \multicolumn{1}{c}{ Características } \\
\hline SCM & $\begin{array}{l}\text { Supply Chain } \\
\text { Management }\end{array}$ & $\begin{array}{l}\text { Gerenciamento } \\
\text { da Cadeia de } \\
\text { Suprimento }\end{array}$ & $\begin{array}{l}\text { Suporta o planejamento e a produção, provendo } \\
\text { informações como onde estão as matérias primas dos } \\
\text { produtos a serem fabricados, a situação da compra de } \\
\text { insumos o cronograma de execução e entrega dos } \\
\text { mesmos }\end{array}$ \\
\hline CRM & $\begin{array}{l}\text { Customer } \\
\text { Relationship } \\
\text { Management }\end{array}$ & $\begin{array}{l}\text { Gerenciamento } \\
\text { do } \\
\text { Relacionamento } \\
\text { com Clientes }\end{array}$ & $\begin{array}{l}\text { Facilitador do gerenciamento dos contatos efetuados } \\
\text { com os clientes, possibilitando criar uma base de } \\
\text { dados específica sobre os serviços ou produtos } \\
\text { relacionados a cada cliente. }\end{array}$ \\
\hline
\end{tabular}




\begin{tabular}{c|l|l|l} 
SEM & $\begin{array}{l}\text { Supplier } \\
\text { Relationship } \\
\text { Management }\end{array}$ & $\begin{array}{l}\text { Gerenciamento } \\
\text { do } \\
\text { Relacionamento } \\
\text { com } \\
\text { Fornecedores }\end{array}$ & $\begin{array}{l}\text { De forma idêntica ao CRM, efetua a gestão dos } \\
\text { contatos realizados com os fornecedores, atualizando } \\
\text { informações concernentes a processos de compra e } \\
\text { entrega de matérias primas. }\end{array}$ \\
\hline PLM & $\begin{array}{l}\text { Product } \\
\text { Mifecycle }\end{array}$ & $\begin{array}{l}\text { Gerenciamento } \\
\text { do Ciclo de Vida } \\
\text { do Produto }\end{array}$ & $\begin{array}{l}\text { Contempla o gerenciamento do ciclo de vida do } \\
\text { produto fabricado pela empresa, desde a sua } \\
\text { concepção, passando pelo projeto, prototipação, } \\
\text { fabricação e testes de qualidade. Está ligado à } \\
\text { capacidade de inovação das empresas. }\end{array}$ \\
\hline ELM & $\begin{array}{l}\text { Employee } \\
\text { Lifecycle } \\
\text { Management }\end{array}$ & $\begin{array}{l}\text { Gerenciamento } \\
\text { do Ciclo de Vida } \\
\text { Funcional dos } \\
\text { Empregados }\end{array}$ & $\begin{array}{l}\text { Sistema relacionado ao gerenciamento de todas as } \\
\text { etapas profissionais dos empregados da organização, } \\
\text { iniciando com a contratação, treinamento, efetivação, } \\
\text { promoções e desligamento da empresa. }\end{array}$ \\
\hline CPM & $\begin{array}{l}\text { Corporate } \\
\text { Performance } \\
\text { Management }\end{array}$ & $\begin{array}{l}\text { Gerenciamento } \\
\text { dos Resultados } \\
\text { da Corporação }\end{array}$ & $\begin{array}{l}\text { É descrito como o "guarda-chuva" que contempla as } \\
\text { metodologias, métricas, processos e sistemas } \\
\text { utilizados para monitorar e gerenciar o desempenho } \\
\text { dos negócios da companhia, gerando visões de todas } \\
\text { as perspectivas do negócio. }\end{array}$ \\
\hline
\end{tabular}

Fonte: Adaptado de Moller, C. (2005). ERP II: A conceptual model for next-generation enterprise systems. Journal of Enterprise Information Management, 18(4), 483-497.

A partir da descrição das características destes aplicativos ditos corporativos, nota-se que os mesmos possuem canais naturais de integração de dados com os ERP's. De certa forma, após a maturação do modelo ERP mais Sistemas Corporativos, formando assim um leque de aplicativos integrados, o ERP II, já em uma fase posterior a este modelo inicial, procura eliminar as interfaces entre estes sistemas, criando uma maior integridade da base de dados.

De fato, ao constatarmos que o ERP II abarca funcionalidades que originariamente pertenciam a outras categorias de aplicativos organizacionais, o principal ganho relacionado ao desenvolvimento de tais aplicativos reside na constatação de que maior número de funcionalidades passou a estar integrado de forma nativa, eliminado problemas relacionados à existência de interfaces e incrementando o desempenho destes aplicativos. Assim, tanto a manutenção como a utilização da totalidade de funcionalidades do ERP II passam a ser mais simples, gerando uma melhor relação benefício versus custo de aquisição e de manutenção.

A terceira camada é composta por aplicativos cujas funcionalidades permitem a integração efetiva de dados e de informações entre o ambiente interno e externo à empresa. De acordo com a estruturação sugerida por Moller (2005), estes sistemas possuem as características expressas na Tabela 2: 


\section{Tabela 2}

Características dos sistemas colaborativos

\begin{tabular}{c|c|c|l}
\hline Sigla & Definição & Tradução & \multicolumn{1}{c}{ Características } \\
\hline B2B & $\begin{array}{c}\text { Business-to- } \\
\text { Business }\end{array}$ & $\begin{array}{c}\text { Negócio para } \\
\text { Negócio }\end{array}$ & $\begin{array}{l}\text { Promove maior eficiência dos processos de compra, } \\
\text { através da automação e descentralização das rotinas } \\
\text { de comunicação entre os integrantes da cadeia de } \\
\text { suprimentos. }\end{array}$ \\
\hline B2C & $\begin{array}{c}\text { Business-to- } \\
\text { Costumer }\end{array}$ & $\begin{array}{c}\text { Negócio para } \\
\text { Cliente }\end{array}$ & $\begin{array}{l}\text { Denota a prática de vendas a clientes através de } \\
\text { transações eletrônicas baseadas em catálogos. O } \\
\text { ERP tem a função de registrar e ordenar as } \\
\text { transações, enquanto um aplicativo web realiza o } \\
\text { interfaceamento com o cliente final. }\end{array}$ \\
\hline B2E & $\begin{array}{c}\text { Business-to- } \\
\text { Employee }\end{array}$ & $\begin{array}{c}\text { Negócio para } \\
\text { Empregados }\end{array}$ & $\begin{array}{l}\text { Propicia a utilização remota dos recursos de TI da } \\
\text { organização por parte de seus funcionários, tanto } \\
\text { para realizar funções administrativas como } \\
\text { direcionadas às atividades-fim da empresa. }\end{array}$ \\
\hline EAI & $\begin{array}{c}\text { Enterprise } \\
\text { Application } \\
\text { Integration }\end{array}$ & $\begin{array}{c}\text { Integração dos } \\
\text { Aplicativos } \\
\text { Empresariais }\end{array}$ & $\begin{array}{l}\text { Também denominado de extranet, constitui-se de } \\
\text { uma integração entre várias plataformas de TI, } \\
\text { fomentando a colaboração eletrônica entre as } \\
\text { empresas. }\end{array}$ \\
\hline
\end{tabular}

Fonte: adaptado de Moller, C. (2005). ERP II: A conceptual model for next-generation enterprise systems. Journal of Enterprise Information Management, 18(4), 483-497.

Diante das definições que procuram descrever o ERP II como um aplicativo ou um somatório de aplicativos de várias naturezas e com diversas funcionalidades diferentes, ainda que complementares, nota-se que quanto mais externa for a camada em que se encontram estes aplicativos (Figura 5), mais complexa torna-se a absorção destas funcionalidades por um único aplicativo.

Esta visão, com relação à aplicação dos sistemas ERP como instrumento consolidador, permitem visualizar as estruturas dos ERPs como a grande base de dados corporativa dos sistemas de informação das empresas. Tal tendência significa, de acordo com Caruso e Johnson (1999), que os sistemas ERP constituem a "espinha dorsal" dos sistemas de informação das empresas. Consequentemente, como visto na definição do ERP II, esses sistemas estão estabelecendo uma plataforma comum para as várias tecnologias atualmente empregadas pelas organizações (Venkatraman \& Henderson, 1998).

Para analisar os benefícios intangíveis do ERPII, deve-se ponderar que a tecnologia em si não produz diferencial competitivo sustentável, uma vez que pode ser imitada com relativa facilidade pelos concorrentes. Por outro lado, as capacidades organizacionais voltadas para a criação de valor de forma repetida e consistente, por meio do uso de seus ativos tecnológicos, representam padrões 
idiossincráticos difíceis de imitar, pois renovam-se ao longo do tempo (Lu, \& Jinghua, 2012).

A literatura apresenta várias linhas de abordagem quanto à definição e mecanismos de formação das capacidades dinâmicas. Como limitação desta pesquisa, foram adotadas duas abordagens proeminentes, a saber: contingencial e integrativa (Camargo \& Meirelles, 2012).

\subsection{Vantagens contingenciais em continuidade}

Uma abordagem das capacidades dinâmicas tem caráter contingencial (Eisenhardt \& Martin, 2000). Nesta perspectiva, depreende-se que o desempenho competitivo é resultado de vantagens temporárias, criadas em continuidade, sendo que as capacidades dinâmicas podem ser observadas embutidas (ou agregadas) em rotinas mais simples, tendo seu principal fator de impulsão relacionado com as funções de aprendizagem organizacional. A companhia adquire habilidades em criar configurações de recursos com mais rapidez e maior efetividade do que seus concorrentes (Schreyögg \& Kliesch-Eberl, 2005).

Esta abordagem define as capacidades dinâmicas como sendo rotinas simples, experimentais, baseadas em conhecimentos tácitos da companhia (ad-hoc) e interativas (não lineares), baseadas em processos frágeis e sem potencial de previsibilidade, desenvolvidas pela recombinação dos recursos por meio do comportamento organizacional, bem como da criação do conhecimento em tempo real para resolução de uma determinada situação (Ambrosini \& Bowman, 2009; Schreyögg \& Kliesch-Eberl, 2005).

Baseados no enfoque contingencial, apresentam-se os estudos de Pavlou (2004), Pavlou e El Sawy (2006) e Pavlou e El Sawy (2011) que analisaram o impacto das capacidades dinâmicas de aprendizagem na formação de rotinas operacionais de sustentação dos sistemas de informação (Sls). Esses estudos identificam algumas capacidades de $\mathrm{TI}$ como sendo capacidades dinâmicas e denotaram seu impacto positivo no desempenho organizacional. Essas capacidades fizeram parte do constructo que estabeleceu a modelagem desenvolvida na pesquisa. 


\subsection{Prospecção e tratamento de oportunidades do mercado}

Teece (1997) introduziu o enfoque integrativo das capacidades dinâmicas. O ponto central desta abordagem enfatiza as ações gerenciais e a criação de clusters de missão crítica. Essas áreas têm função de construir, estabilizar e reconfigurar as rotinas operacionais, de modo a satisfazer os requisitos do ambiente de negócios. Como resultado obtém-se o desenvolvimento de produtos tangíveis ou intangíveis, no caso o ERPII.

Em complemento, Teece (2011) sustenta que a formação das capacidades dinâmicas se baseia no relacionamento da empresa com o mercado em que ela está inserida. Esses processos são definidos como: (i) percepção das oportunidades do ambiente (sensing), que consiste em pesquisar as tecnologias e os ecossistemas, prospecção de fornecedores, necessidades dos clientes, comportamento dos competidores; (ii) o tratamento das oportunidades conforme são percebidas, (seizing) por meio de moldagem de processos internos, desenvolvimento das competências tecnológicas, por meio de protocolos de tomada de decisão e reconhecimento dos limites organizacionais; (iii) capacidade de recombinar os recursos e competências existentes (reconfiguring), redesenho do modelo de negócios, rotinas e iniciativas de descentralização visando o aumento da flexibilidade.

\subsection{Mensuração do valor de uso do ERPII}

Para mensurar a percepção do valor de uso ERPII, este trabalho usou o modelo teórico-empírico proposto por Delone e Mclean (1992), dedicado à análise dos fatores de sucesso na implementação dos sistemas de informação. Os autores revisitaram seu trabalho original em Delone e Mclean (2003), adicionando 150 publicações baseadas em sua obra de 1992. Este volume de dados garante uma robustez ao modelo conceitual proposto. Esses fatores também foram identificados por outros autores, como se segue:

a) Qualidade do sistema: conforme Delone e Mclean (2003) representa características positivas dos sistemas integrados: usabilidade, disponibilidade, confiabilidade, adaptabilidade, e tempo de resposta. 
b) Qualidade de uso: de acordo com Jain (2010), corresponde à capacidade dos usuários em usar o ERPII de maneira extensiva no decorrer do tempo, explorando novas funcionalidades não apresentadas em sua fase de implementação, tendo como pressuposto a apropriação dessas novas formas de uso do sistema.

c) Qualidade da informação: para Canhette (2004), este fator consiste na capacidade do ERPII em fornecer informações analíticas valiosas se usadas no processo de tomada de decisão, e na capacidade de tomar decisões baseadas em evidências em detrimento de opiniões.

\subsection{Modelo da pesquisa}

Para Bunge (1974) existe uma dicotomia entre o conhecimento teórico e o empírico. Se por um lado as teorias são estruturadas por meio de abstrações, por outro lado os dados empíricos, mais próximos do mundo real, carecem de sentido lógico para a compreensão. No intuito de reconciliar os conceitos com as mensurações, tem-se a importância da criação de modelos, que estabelecem relações sujeitas a inferência.

Esta abordagem se aplica ao desenvolvimento das capacidades dinâmicas relacionadas com benefícios intangíveis à organização. Essas não são observáveis por meio de evidências materiais, mas pela análise do discurso e dos elementos tácitos embutidos nas rotinas organizacionais. Um método adequado é avaliar o nível de aderência dos respondentes a uma pesquisa de campo, conforme aplicado neste trabalho (Wang, \& Ahmed, 2007).

O modelo proposto para este estudo, conforme descrito na Figura 3, tem o objetivo de articular as variáveis relativas aos fatores de formação das capacidades dinâmicas, com os indicadores de percepção de valor de uso do ERPII. 
Recombinação de recursos (ciclos de atualização impulsionados por aprendizagem)

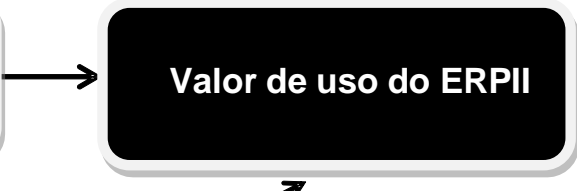

Modelo 1: Abordagem contingencial

Prospecção e tratamento de oportunidades do mercado

Modelo 2: Abordagem integrativa

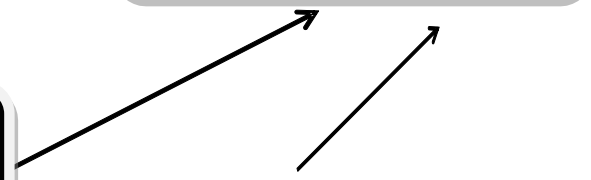

Qualidade do Sistema

Qualidade do Uso

Qualidade da Informação

Figura 3 - Modelo da Pesquisa: Abordagens das capacidades dinâmicas e seu impacto no Valor de Uso do ERPII.

Fonte: Dados da Pesquisa.

Os constructos do estudo são apresentados na Tabela 3. O primeiro conjunto de variáveis independentes está calcado na abordagem contingencial. Neste entendimento, as capacidades dinâmicas impactam o desempenho organizacional pela obtenção de vantagens temporárias, criadas em continuidade, observadas em rotinas mais simples (commonalities ou 'best practices'). Tais rotinas são incentivadas pela aprendizagem e são predecessoras das capacidades dinâmicas. Como resultado de tais interações, os autores enfatizam o aumento da transferência de conhecimento por meio da aprendizagem (Eisenhardt, \& Martin, 2000).

\section{Tabela 3}

Constructos delineadores do Instrumento de Pesquisa

VARIÁVEIS

\begin{tabular}{r}
\hline \hline \\
Incentivo ao \\
ambiente de \\
aprendizagem
\end{tabular}

conhecimento

tácito em

explícito
EXPLICAÇÕES

BASE TEÓRICA

\section{Abordagem Contingencial}

A articulação do conhecimento é papel da alta gerência, com mecanismos de incentivo ao compartilhamento de experiência, discussões grupais, e programas de sugestões de melhorias.

A formação das CDs depende de reconfiguração de recursos em busca do desempenho, pela acumulação de experiência tácita e codificação posterior do conhecimento explícito.
Teece (1997), Ambrosini E Bowman (2009), Zollo e Winter (2002), Eisenhardt e Martin (2000), Wang e Ahmed (2007), Nonaka (1994), Schreyögg e Kliesch (2005), Pavlou (2004), Pavlou e El Sawy (2006), Jain (2008) 


\begin{tabular}{rl|l}
$\begin{array}{r}\text { Cultura de } \\
\text { melhoria } \\
\text { contínua }\end{array}$ & $\begin{array}{l}\text { Empresas que possuem cultura } \\
\text { organizacional disposta a mudanças } \\
\text { continuas impulsionam as CDs através } \\
\text { da aprendizagem. }\end{array}$ & $\begin{array}{l}\text { Jain (2008), Pavlou (2004), } \\
\text { Pavlou e El Sawy (2006), } \\
\text { Pavlou e El Sawy (2011), } \\
\text { Bhatt e Grover (2005) }\end{array}$ \\
\hline $\begin{aligned} \text { Capacitação } \\
\text { para uso do }\end{aligned}$ & $\begin{array}{l}\text { É papel da TI incentivar treinamentos no } \\
\text { ERPIII e em processos de negócios } \\
\text { envolvidos. Isso evita a dissipação das } \\
\text { CDs de uso do sistema. }\end{array}$ & $\begin{array}{l}\text { Clark, Jones e Zmud (2009), } \\
\text { Beachboard (2005), Bullon } \\
(2009), \text { Sambamurthy e Zmud } \\
(2000) ; \text { Byrd, Lewis e Turner } \\
(2004) ;\end{array}$ \\
\hline
\end{tabular}

\begin{tabular}{|c|c|c|}
\hline \multicolumn{3}{|c|}{ Abordagem Integrativa } \\
\hline $\begin{array}{r}\text { Cultivo de } \\
\text { novas } \\
\text { tecnologias }\end{array}$ & $\begin{array}{l}\text { O acúmulo da tecnologia para potencial } \\
\text { futuro incentiva visão ex-ante, favorável } \\
\text { ao desenvolvimento das CDs em } \\
\text { ambientes de incerteza. }\end{array}$ & $\begin{array}{l}\text { Josemin (2010), Ciborra } \\
\text { (1997), Bhatt e Grover (2005) }\end{array}$ \\
\hline $\begin{array}{r}\text { Prospecção } \\
\text { mercado } \\
\begin{array}{r}\text { Tratamento } \\
\text { mercado }\end{array}\end{array}$ & $\begin{array}{l}\text { Estabelecer rotinas de interação com o } \\
\text { mercado para atualização do ERPII } \\
\text { corresponde à CDs de natureza } \\
\text { transformacional. É papel da TI } \\
\text { converter oportunidades em } \\
\text { atualizações do ERPII. }\end{array}$ & $\begin{array}{l}\text { Schreyögg e Kliesch (2005), } \\
\text { Teece (1997), Teece (2011), } \\
\text { Cyryno (2000), Josemin } \\
\text { (2010), Teece (2007), } \\
\text { Nedzinskas (2014), Helfat } \\
(1997), \text { Zahra e George } \\
\text { (2002), Wang e Ahmed } \\
(2007),\end{array}$ \\
\hline $\begin{array}{r}\text { Conversão } \\
\text { necessidades } \\
\text { em atualizações }\end{array}$ & $\begin{array}{l}\text { A área de TI deve atuar próxima às } \\
\text { áreas de negócios para compreender } \\
\text { necessidades, convertendo-as em } \\
\text { soluções sistêmicas. }\end{array}$ & $\begin{array}{l}\text { Biancolino, Maccari e Pereira } \\
\text { (2013), Jain (2008), Mizik e } \\
\text { Jacobson (2003), Souza e } \\
\text { Zwicker (2007), Byrd, Lewis e } \\
\text { Turner (2004) }\end{array}$ \\
\hline
\end{tabular}

\section{Valor de uso do ERP II}

\begin{tabular}{|c|c|c|}
\hline $\begin{array}{c}\text { Valor do } \\
\text { sistema }\end{array}$ & $\begin{array}{l}\text { Como fator de qualidade, o ERPII deve } \\
\text { possuir altos níveis de disponibilidade, } \\
\text { tempo de resposta, confiabilidade. }\end{array}$ & $\begin{array}{l}\text { Delone e Mclean (1992) } \\
\text { Delone e Mclean (2002) } \\
\text { Clark, Jones e Zmud (2009) } \\
\text { Canhete (2004) }\end{array}$ \\
\hline Valor de uso & $\begin{array}{l}\text { Como fator de qualidade, os usuários } \\
\text { devem fazem uso extensivo do ERPII, } \\
\text { buscando novas funcionalidades. }\end{array}$ & $\begin{array}{l}\text { Delone e Mclean (1992) } \\
\text { Delone e Mclean (2002) } \\
\text { Clark, Jones e Zmud (2009) } \\
\text { Canhete (2004) }\end{array}$ \\
\hline $\begin{array}{r}\text { Valor da } \\
\text { informação }\end{array}$ & $\begin{array}{l}\text { Como fator de qualidade, o ERPII deve } \\
\text { fornecer aos gerentes informações para } \\
\text { tomada de decisão. }\end{array}$ & $\begin{array}{l}\text { Delone e Mclean (1992) } \\
\text { Delone e Mclean (2002) } \\
\text { Clark, Jones e Zmud (2009) } \\
\text { Canhete (2004) }\end{array}$ \\
\hline
\end{tabular}

Fonte: Dados da Pesquisa.

O segundo grupo de variáveis independentes observa o enfoque integrativo e reforça o papel gerencial no incentivo à formação das capacidades dinâmicas, representadas por meio dos clusters de missão crítica. Estes são compostos por 
equipes multidisciplinares, responsáveis por potencializar as atividades coletivas necessárias para estabilizar e reconfigurar as rotinas organizacionais, de modo a satisfazer as mudanças do ambiente de negócios. Os clusters são acumuladores de capacidades dinâmicas, e este trabalho considera-os estabelecidos nas áreas de $\mathrm{TI}$ das organizações (Schreyögg \& Kliesch-Eberl, 2005; Teece, 2012).

Esta abordagem enfatiza a habilidade em explorar oportunidades de inovação presentes do mercado, com pesquisa e desenvolvimento de novas tecnologias, prospecção de fornecedores e concorrentes. Essa capacidade dinâmica envolve a redefinição de modelos de negócios, descentralização, aumento de flexibilidade e resposta aos clientes que usam ferramentas tecnológicas. Com efeito, as capacidades dinâmicas influenciam positivamente a evolução dos ativos tangíveis e intangíveis, neste caso, atualizações das funcionalidades e módulos do ERPII (Vasconcelos \& Cyryno, 2000; Teece, 2012).

Uma variável adicional foi agregada ao modelo, baseada em Josemin (2010) a partir de Ciborra (1997) e Bhatt e Grover (2005). O autor identificou o cultivo da tecnologia e seu acúmulo superestimado como uma capacidade dinâmica. Isto potencializa impactos da tecnologia em benefícios organizacionais.

\section{Metodologia}

\subsection{Caracterização da pesquisa, organização e amostra}

A pesquisa é de natureza descritiva e explicativa; analisa o problema baseado nas informações coletadas de uma amostra da população, a partir de um escopo de estudo estabelecido de maneira estruturada. O escopo amostral é composto por profissionais da área de tecnologia da informação (TI) que atuam em organizações de grande porte no cenário brasileiro. Quanto ao tipo da amostra, foi empregada a seleção por acessibilidade, baseada na experiência prévia de um dos autores. Esta maneira de seleção de respondentes traz a vantagem de identificar aqueles mais adequados às condições estabelecidas (Magalhães \& Hill, 2005).

\subsection{Instrumento de pesquisa}

O questionário usado no instrumento de pesquisa foi estruturado em escala Likert de 10 posições. Em sua montagem, foram usadas 10 perguntas fechadas, 
baseadas nas variáveis do constructo, e duas abertas (ramo e cargo). As questões são apresentadas aos respondentes na mesma ordem de exibição e com o mesmo número de opções (Magalhães \& Hill, 2005).

A pesquisa retornou 374 questionários completados. De acordo com o cálculo do Alpha de Cronbach, conforme Tabela 4, as escalas dos constructos atingiram níveis aceitáveis $(0,9>\alpha \geq 0,8)$ quanto à confiabilidade do instrumento (Hair, 1998).

\section{Tabela 4}

Alpha de Cronbach

\begin{tabular}{l|c|c|c|c}
\multicolumn{1}{c}{ Constructo } & \multirow{2}{*}{$\begin{array}{c}\text { N } \\
\text { Excluído }\end{array}$} & Assertivas & $\begin{array}{c}\text { Alpha de } \\
\text { Cronbach }\end{array}$ \\
\hline \hline Capacidades Dinâmicas - abordagem contingencial & & & 4 & 0,866 \\
\hline Capacidades Dinâmicas - abordagem integrativa & \multirow{3}{*}{374} & 0 & 4 & 0,875 \\
\hline Valor de Uso do ERPII & & & 3 & 0,710 \\
\hline
\end{tabular}

Fonte: Dados da Pesquisa.

O questionário foi submetido à revisão de pares com 10 (dez) respondentes por meio de um pré-teste. $O$ resultado apontou alterações para aumentar a clareza de determinadas questões e a mudança de alguns termos para linguagem de mercado.

\subsection{Procedimento de coleta e tratamento dos dados}

O questionário foi construído em plataforma eletrônica, por meio de uma ferramenta de pesquisas padrão de mercado, e o link foi enviado por e-mail aos participantes da pesquisa. Esta estratégia garantiu a otimização de custos, velocidade, abrangência, gerenciamento em tempo real das respostas e extração do banco de dados (SPSS). Para os respondentes, apresentou facilidade de gerenciamento do tempo e local apropriado para resposta (Malhotra \& Galletta, 1999).

Os dados coletados foram tratados antes da realização dos testes estatísticos, e os outliers foram eliminados por meio do método de distância de Mahalonobis conforme relatado em Reimann, Filzmoser, Garrett e Dutter (2010).

Os pressupostos para os testes de regressão linear múltipla foram satisfeitos. Quanto à independência, os dados não apresentaram altas correlações entre os pares de assertivas. Com a execução de validações VIF (variance inflation factor) foi 
afastada a probabilidade de multicolinearidade entre os grupos de variáveis $(>10,0)$. As suposições dos resíduos foram detectadas por meio de gráficos de resíduos ajustados, e não houve a observância de heteroscedasticidade para os dados da amostra (Hair, 1998).

\section{Apresentação e Discussão dos Resultados}

A Tabela 5 detalha as estatísticas descritivas (média, desvio padrão) e as correlações entre as variáveis analisadas na pesquisa. Conforme o modelo proposto, deve-se avaliar as correlações entre as 8 variáveis das capacidades dinâmicas (em suas duas abordagens) em contraposição aos 3 indicadores de percepção de valor de uso do ERPII. Neste aspecto, o resultado mostrou correlações de moderada (> $0,3)$ para forte $(>0,5)$ entre tais combinações. Houve significância $(p<0,01)$ para todas as correlações (Cohen,1992).

A variável com maior influência nos indicadores de valor de uso do ERPII (VS, $\mathrm{VU}, \mathrm{VI}$ ) foi a prospecção do mercado para novas soluções de tecnologia (PM), com correlações de 0,379; 0,403 e 0,505. Outras correlações importantes foram identificadas: observou-se um impacto positivo de 0,435 da variável conversão das necessidades de negócios (NN) no indicador de valor do sistema (confiabilidade) (VS). Por sua vez, a variável cultivo da tecnologia (CT) e prospecção do mercado (PM) influenciaram positivamente os indicadores de valor de uso (uso extensivo) (VU) do sistema de gestão (0,406 e 0,403 respectivamente).

\section{Tabela 5}

Estatísticas Descritivas e Correlações entre as variáveis da Pesquisa

\section{Correlações}

\section{Abordagem Contingencial Abordagem Integrativa $\quad$ Valor Uso ERPII}

\begin{tabular}{|c|c|c|c|c|c|c|c|c|c|c|c|c|}
\hline Média & DP & $\mathrm{AA}$ & TE & MC & CS & CT & PM & TO & NN & VS & VU & VI \\
\hline \multicolumn{13}{|c|}{ Variável: Incentivo Ambiente aprendizagem (AA) } \\
\hline 5,50 & 2,508 & 1 & & & & & & & & & & \\
\hline \multicolumn{13}{|c|}{ Variável: Conversão conhecimento tácito em explícito (TE) } \\
\hline 6,69 & 2,267 &, $453^{* *}$ & 1 & & & & & & & & & \\
\hline \multicolumn{13}{|c|}{ Variável: Cultura de melhoria contínua (MC) } \\
\hline 6,08 & 2,547 &, $482^{\star *}$ & ,288** & 1 & & & & & & & & \\
\hline
\end{tabular}


\begin{tabular}{ll|lll}
5,83 & 2,559 & $, 516^{* *}, 347^{\star *}, 408^{\star *} \quad 1$
\end{tabular}

Variável: Cultivo de novas tecnologias (CT)

$6,492,475\left|, 486^{\star *}, 351^{\star *}, 557^{\star *}, 464^{\star *}\right| 1$

Variável:Prospecção mercado; atualização do ERPII (PM)

\begin{tabular}{ll|lll|ll}
6,37 & 2,520 & $, 422^{\star *}, 440^{\star \star}, 444^{\star *}, 442^{\star *}$ &, $609^{\star \star}$ & 1
\end{tabular}

Variável:Tratamento mercado; atualização do ERPII (TO)

\begin{tabular}{ll|lll|l|l}
6,33 & 2,508 & $, 443^{\star *}, 415^{\star *}, 349^{\star \star}, 472^{\star *}$ & $, 604^{\star \star}, 701^{* \star}$ \\
\hline
\end{tabular}

Variável:Conversão necessidades em atualizações (NN)

\begin{tabular}{ll|lll|llll}
7,17 & 1,999 & $, 396^{* *}, 415^{* *}, 339^{* *}, 483^{* *}$ & $, 444^{* *}, 451^{* *}, 421^{* *}$ & 1
\end{tabular}

Variável:Valor do sistema; confiabilidade (VS)

\begin{tabular}{ll|lll|lll|l}
7,85 & 2,096 & $194^{* *}, 280^{\star *}$ & $, 290^{\star *}, 284^{* *}$ & $, 305^{\star *}, 379^{* *}, 371^{* *}, 435^{\star *}$ & 1
\end{tabular}

Variável:Valor de uso; uso extensivo (VU)

\begin{tabular}{ll|lll|lll|l}
6,31 & 2,475 & $, 350^{* *}, 312^{* *}, 327^{* *}, 405^{* *}$ & $, 406^{\star *}, 403^{* *}, 303^{* *}, 366^{* *}$ &, $385^{\star *}$ & 1
\end{tabular}

Variável:Valor da informação; tomada de decisão (VI)

\begin{tabular}{c|c|cccc|cccc|ccc}
6,96 & 2,331 &, $396^{\star *}$ &, $408^{\star *}$ &, $361^{* *}$ &, $470^{\star *}$ &, $498^{* *}$ &, $505^{\star *}$ &, $418^{* *}$ &, $489^{* *}$ &, $541^{* *}$ &, $486^{* *}$ & 1 \\
\hline Média & DP & AA & TE & MC & CS & CT & PM & TO & NN & VS & VU & VI \\
\hline
\end{tabular}

Nota. ${ }^{* *}$ Correlação significativa no nível 0,01 (bilateral)

Fonte: Dados da Pesquisa.

A Tabela 6 apresenta os resultados dos testes de regressão linear múltipla para o grupo de variáveis das capacidades dinâmicas sob a abordagem contingencial. Como variáveis independentes temos conversão do conhecimento tácito em explícito (TE), cultura de melhoria contínua (MC) e capacitação para uso do ERPII (CS). A análise dos resultados $\left(\mathrm{R}^{2}\right)$ corrobora o apresentado no teste de correlações, e denota um tamanho de impacto entre médio (>13\%) e grande (>26\%) (Cohen, 1992).

\section{Tabela 6}

Resultados das Equações de Regressão Linear Múltipla

\section{Modelo 1 - Contingencial}

\begin{tabular}{|c|c|c|c|c|c|}
\hline VD & $\begin{array}{c}\text { Teste } \\
\text { Estatístico }\end{array}$ & $\mathbf{R}\left(\mathbf{R}^{2}\right)$ & VI & $\beta$ & sig \\
\hline $\begin{array}{l}\text { Valor do Sistema } \\
\text { (confiabilidade, resposta, } \\
\text { disponibilidade) }\end{array}$ & $\begin{array}{l}F=10,37 \\
p=0,000\end{array}$ & $\begin{array}{c}0,430 \\
(0,185)\end{array}$ & $\begin{array}{l}\text { Conversão do conhecimento tácito } \\
\text { Cultura de melhoria contínua }\end{array}$ & $\begin{array}{l}0,183 \\
0,154\end{array}$ & $\begin{array}{l}0,020 \\
0,007\end{array}$ \\
\hline $\begin{array}{l}\text { Valor de Uso (uso } \\
\text { extensivo, novas } \\
\text { funcionalidades) }\end{array}$ & $\begin{array}{l}F=14,86 \\
p=0,000\end{array}$ & $\begin{array}{c}0,496 \\
(0,246)\end{array}$ & Cultura de melhoria contínua & 0,135 & 0,014 \\
\hline $\begin{array}{l}\text { Valor da Informação } \\
\text { (tomada de decisão) }\end{array}$ & $\begin{array}{l}F=21,93 \\
p=0,000\end{array}$ & $\begin{array}{c}0,326 \\
(0,311)\end{array}$ & $\begin{array}{l}\text { Conversão do conhecimento tácito } \\
\text { Cultura de melhoria contínua } \\
\text { Capacitação para uso do sistema }\end{array}$ & $\begin{array}{l}0,202 \\
0,109 \\
0,190\end{array}$ & $\begin{array}{l}0,000 \\
0,023 \\
0,005\end{array}$ \\
\hline
\end{tabular}

Fonte: Dados da Pesquisa, 2017. 
Por sua vez, a Tabela 7 sintetiza as relações entre o grupo de variáveis das capacidades dinâmicas baseadas na visão integrativa/gerencial. As variáveis independentes são: prospecção do mercado para oportunidades de atualização do ERPII (PM), conversão das necessidades de negócios em atualizações do ERPII (NN), tratamento das oportunidades de mercado (TO) e cultivo de novas tecnologias (CT).

\section{Tabela 7}

Resultados das Equações de Regressão Linear Múltipla

\begin{tabular}{|c|c|c|c|c|c|}
\hline \multicolumn{6}{|c|}{ Modelo 2 - Integrativo } \\
\hline VD & $\begin{array}{c}\text { Teste } \\
\text { Estatístico }\end{array}$ & $\mathbf{R}\left(\mathbf{R}^{2}\right)$ & VI & $\beta$ & sig \\
\hline $\begin{array}{l}\text { Valor do Sistema } \\
\text { (confiabilidade, resposta, } \\
\text { disponibilidade) }\end{array}$ & $\begin{array}{c}F=9,58 \\
p=0,000\end{array}$ & $\begin{array}{c}0,535 \\
(0,286)\end{array}$ & $\begin{array}{l}\text { Prospecção oportunidades } \\
\text { de mercado } \\
\text { Conversão necessidades de } \\
\text { negócios ERPII }\end{array}$ & $\begin{array}{l}0,134 \\
0,229\end{array}$ & $\begin{array}{l}0,050 \\
0,000\end{array}$ \\
\hline $\begin{array}{l}\text { Valor de Uso (uso } \\
\text { extensivo, novas } \\
\text { funcionalidades) }\end{array}$ & $\begin{array}{l}F=10,14 \\
p=0,000\end{array}$ & $\begin{array}{c}0,547 \\
(0,299)\end{array}$ & $\begin{array}{l}\text { Prospecção oportunidades } \\
\text { de mercado Tratamento } \\
\text { oportunidades de mercado }\end{array}$ & $\begin{array}{l}0,174 \\
0,143\end{array}$ & $\begin{array}{l}0,013 \\
0,041\end{array}$ \\
\hline $\begin{array}{l}\text { Valor da Informação } \\
\text { (tomada de decisão) }\end{array}$ & $\begin{array}{l}F=17,19 \\
p=0,000\end{array}$ & $\begin{array}{c}0,421 \\
(0,396)\end{array}$ & $\begin{array}{l}\text { Cultivo de novas tecnologias } \\
\text { Prospecção oportunidades } \\
\text { de mercado Conversão } \\
\text { necess. negócios no ERPII }\end{array}$ & $\begin{array}{l}0,164 \\
0,183 \\
0,203\end{array}$ & $\begin{array}{l}0,005 \\
0,002 \\
0,003\end{array}$ \\
\hline
\end{tabular}

Fonte: Dados da Pesquisa, 2017.

A variável que recebeu maior impacto positivo das capacidades dinâmicas foi a de valor do ERPII para a tomada de decisão (VI), e obteve poder preditivo de tamanho de efeito grande (40\%) quando orquestrada pelas variáveis independentes: cultivo de novas tecnologias (CT), prospecção de oportunidades do mercado (PM) e conversão de necessidades de negócios em atualizações do ERPII (NN).

De maneira geral, as capacidades dinâmicas identificadas nas áreas de TI foram importantes preditoras dos índices de mensuração de valor do ERPII para as organizações. O modelo conceitual das capacidades dinâmicas que obteve maior tamanho de efeito nesta relação é o integrativo (Modelo 2), que impõe ênfase no papel gerencial na integração organizacional.

Um primeiro conjunto de capacidades dinâmicas foi identificado como influenciador do valor de uso do ERPII. Tratam-se dos mecanismos de aprendizagem. Neste aspecto as variáveis TE, MC, e CS predizem em $31 \%$ o valor 
de informação, a saber, o uso estratégico do ERPII para tomada de decisão (VI). Para Ambrosini e Bowman (2009), as capacidades dinâmicas não se formam de maneira autônoma, mas surgem, por um outro lado, como resultado de várias ações multidisciplinares voltadas ao incentivo à aprendizagem organizacional.

Quanto a esses achados, Pavlou (2004), Pavlou e El Sawy (2006) e Pavlou e El Sawy (2011) explicam que as capacidades dinâmicas influenciam positivamente as rotinas organizacionais de aprendizagem ligadas à sustentação e transformação dos Sistemas de Informação, e que por sua vez influenciam os níveis de percepção de valor destes. Isso se dá especialmente considerando o contexto de altas taxas de mudanças.

Os achados denotam coerência com a literatura, que sustenta que a formação das capacidades dinâmicas não ocorre de maneira autômata, mas incentivada pelas ações e pelo ambiente, com enfoque na aprendizagem organizacional (Ambrosini \& Bowman, 2009).

Já um segundo conjunto de capacidades dinâmicas é voltado à prospecção, cultivo e tratamento das oportunidades de negócios, convertendo-as em atualizações do ERPII. Essas variáveis combinadas tem o poder preditivo de $40 \%$ para a variável de valor da informação do ERPII para tomada de decisão (VI). Teece (2012) identificou como influenciadoras do desempenho organizacional essas capacidades dinâmicas voltadas para percepção (sensing) das oportunidades de mercado do ERPII, bem como a efetividade no tratamento dessas oportunidades, convertendo-as em novas atualizações, funcionalidades e sistemas agregados (seizing / transforming).

Tais resultados foram alinhados com os indicadores de mensuração de valor dos sistemas de informação (SIs) e denotam que as capacidades dinâmicas impõem uma percepção positiva dos respondentes quanto ao valor global do ERPII em sua fase de sustentação (Delone \& Mclean, 1992; Clark, Jones, \& Zmud, 2009; Canhette, 2004).

Em conclusão, a investigação observou que os fatores de maior impacto na percepção positiva do valor do ERPII foram aqueles relativos aos mecanismos inovativos e de prospecção do mercado e consequente tratamento das oportunidades, convertendo-as em atualizações do sistema de gestão. Este 
resultado também é coerente com a abordagem integrativa da formação das capacidades dinâmicas (Teece, 2011).

\section{Conclusão}

O trabalho atingiu os objetivos propostos, pois identificou as capacidades dinâmicas que influenciam positivamente a manutenção do valor de uso do ERPII em sua fase de sustentação. Por conseguinte, conseguiu mensurar os níveis de influência dessas capacidades. Observou-se que, de maneira geral, o valor do uso analítico do ERPII para tomada de decisão foi a variável que recebeu maior impacto positivo quando orquestrada pelas capacidades dinâmicas. Nesta sequência, tem-se impacto, porém de menor tamanho de efeito, nos indicadores de uso extensivo do ERPII e valor deste sistema (confiabilidade, rapidez, estabilidade).

A pesquisa escolheu sua amostra de respondentes dentro do ambiente da TI nas organizações pesquisadas. Sendo assim, serve como uma análise da percepção desses profissionais quanto à área em que eles atuam. As informações das inferências estatísticas apontam uma evolução da maneira de trabalho da TI na sustentação do ERPII, com aumento do enfoque em reagir às mudanças constantes do ambiente de negócios. Isto foi denotado na identificação de capacidades dinâmicas encontradas neste departamento e seu consequente impacto na sustentação dos sistemas de gestão.

Este cenário também caracteriza a limitação da pesquisa e abre espaço para aplicação deste questionário a respondentes dos demais departamentos nas organizações. Pode-se então analisar os resultados em contraposição ( $\mathrm{Tl}$ e outros departamentos clientes do ERPII). Isto abriria lacunas para a identificação de outras perspectivas de interpretação do papel deste importante setor na sustentação do ERPII.

\section{Referências}

Ambrosini, V., \& Bowman, C. (2009). What are dynamic capabilities and are they a useful construct in strategic management? International Journal of Management Reviews, 11(1), 29-49. 
Barney, J. B., \& Clark, D. N. (2007). Resource-based theory: creating and sustaining competitive advantage. Oxford: Oxford University Press.

Bhatt, G. D., \& Grover, V.; (2005). Types of information technology capabilities and their role in competitive advantage: an empirical study. Journal of Management Information Systems, 22(2), 253-277.

Biancolino, C. A. (2010). Valor de uso do ERP e gestão contínua de pós implementação: estudo de casos múltiplos no cenário brasileiro. (Tese de Doutorado. Universidade de São Paulo, São Paulo, SP, Brasil).

Borges, T. N., Parisi, C., \& Gil, A. L. (2005). O controller como gestor da tecnologia da informação: realidade ou ficção? Revista de Administração Contemporânea 9(4) 119-140.

Bunge, M. (1974), Teoria e realidade. São Paulo: Editora Perspectiva.

Camargo, A. A. B. D., \& Meirelles, D. S. (2012). Capacidades dinâmicas: o que são e como identificá-las. Anais do $36^{\circ}$ Encontro Nacional da Associação Nacional de Pós-Graduação em Administração, Rio de Janeiro, RJ, Brasil.

Canhette, C. C (2004). Análise das menções à qualidade da informação em teses e dissertações que relatam impactos do uso de sistemas ERP. (Tese de Doutorado. Universidade de São Paulo, São Paulo, SP, Brasil).

Caruso, D., \& Johnson, R. (1999). The vision thing. Intelligent Enterprise, 18(6).

Ciborra, C. U. (1997) Deconstructing the concept of strategic alignment. Scandinavian Journal of Information Systems, 9(1), 67- 82.

Clark, T. D., Jones, M. C., \& Zmud, R. W. (2009) Post adoptive ERP use behaviors: a dynamic conceptualization. International Conference of the System Dynamics Society, 27(1).

Cohen, J. (1992). A power primer. Psychological Bulletin, 112(1), 155-159.

Delone, W. H., \& Mclean, E. R. (1992). Information systems success: he quest for the dependent variable. Information Systems Research, 3(1), 60-95.

Delone, W. H., \& Mclean, E. R. (2003). The Delone and Mclean model of information systems success: a ten-year update. Journal of Management Information Systems, 19(4), 9-30.

Eisenhardt, K. M., \& Martin, J. A. (2000). Dynamic Capabilities: What are they? Strategic Management Journal, 21(10), 105-112.

Hair J. F. (1988). Multivariate data analysis. New York: Prentice Hall.

Jain, V. (2010). What makes ERP systems to deliver? Impact of post-implementation capabilities on ERP value. ECIS. 
Josemin, G. C. (2010). Capacidades dinâmicas de TI. Anais do 34ํㅡㄹ Encontro Nacional da Associação Nacional de Pós-Graduação em Administração, Rio de Janeiro, RJ, Brasil.

Lu, Z., \& Jinghua, H. (2012). The moderating factors in the relationship between ERP investments and firm performance. The Journal of Computer Information Systems, 53(2), 75-84.

Magalhães, M., \& Hill, A. (2005). Investigação por questionário. Lisboa: Sílabo.

Malhotra, Y., \& Galletta, D. F. (1999). Extending the technology acceptance model to account for social influence: theoretical bases and empirical validation. Systems Sciences. HICSS.

Moller, C. (2005). ERP II: A conceptual model for next-generation enterprise systems. Journal of Enterprise Information Management, 18(4), 483-497.

Norton, A. I. (2013). Ensuring Benefits Realization from ERP II: The CSF phasing model. Journal of Enterprise Information Management, 26(3), 218-234.

Pavlou, P. A. (2004). IT-enabled dynamic capabilities in new product development: building a competitive advantage in turbulent environments. (Tese de Doutorado. University of Southern Califórnia, Califórnia).

Pavlou, P. A., \& El Sawy, O. A. (2006). From IT leveraging competence to competitive advantage in turbulent environments: The case of new product development. Information Systems Research, 17(3), 198-227.

Pavlou, P. A., \& El Sawy, O. A. (2011). Understanding the elusive black box of dynamic capabilities. Decision Sciences, 42(1), 239-273.

Reimann, C., Filzmoser, P., Garrett, R., \& Dutter, R. (2010). Statistical data analysis explained: applied environmental statistics with $R$. New York: Willey.

Schreyögg, G., \& Kliesch-Eberl, M. (2005). How dynamic can organizational capabilities be? towards a dual-process model of capability dynamization. Strategic Management Journal, 28(9), 913-933

Souza, C. A., \& Zwicker, R. (2007). Capacidades e atores na gestão de sistemas ERP: um estudo exploratório entre usuários corporativos do ERP da SAP. Revista de Gestão da Tecnologia e Sistemas de Informação 4(1), 197-216.

Teece, D. J. (1997). Dynamic capabilities and strategic management. Strategic Management Journal, 18(7).

Teece, D. J. (2011). Achieving integration of the business school curriculum using the dynamic capabilities model. Journal of Management Development, 30(5), 499-518.

Teece, D. J. (2012). Dynamic capabilities: routines versus entrepreneurial action. Journal of Management Studies, 49(8). 
Vasconcelos, F. C., \& Cyrino, A. B. (2000). Vantagem competitiva: os modelos teóricos atuais e a convergência entre estratégia e teoria organizacional. Revista de Administração de Empresas, 40(4), 20-37.

Venkatraman, N., \& Henderson, J. C. (1998). Real strategy for virtual organization. Sloan Management Review, 40(1), 33-48.

Wang, C. L., \& Ahmed, P. K. (2007). Dynamic capabilities: a review and research agenda. International Journal of Management Reviews, 9(1), 31-51. 